Revue internationale P.M.E.

Économie et gestion de la petite et moyenne entreprise

\title{
Implantation de petites entreprises en zones aménagées. Territoires à promouvoir ou territoires à prendre?
}

\section{Geneviève Duche et Suzane Savey}

Volume 2, numéro 1, 1989

URI : https://id.erudit.org/iderudit/1007908ar

DOI : https://doi.org/10.7202/1007908ar

Aller au sommaire du numéro

Éditeur(s)

Presses de l’Université du Québec

ISSN

0776-5436 (imprimé)

1918-9699 (numérique)

Découvrir la revue

Citer cette note

Duche, G. \& Savey, S. (1989). Implantation de petites entreprises en zones aménagées. Territoires à promouvoir ou territoires à prendre? Revue internationale P.M.E., 2(1), 71-97. https://doi.org/10.7202/1007908ar

\section{Résumé de l'article}

L'implantation et les modalités de fonctionnement (en particulier la nature des emplois offerts) de l'entreprise doivent s'inscrire dans un consensus minimum d'utilisation des hommes et de l'espace sans lequel il est difficile de créer les synergies nécessaires au développement local.

Il paraît donc essentiel de comprendre les raisons de l'installation des entreprises dans un lieu plutôt qu'un autre, ce qu'elles attendent de l'environnement local mais aussi ce qu'elles lui apportent, et les modalités de la rencontre entre leurs responsables et les élus locaux en charge de définir une politique de développement.

Pour tenter de répondre à ces questions, les auteurs ont travaillé sur quelques exemples de zones aménagées de l'agglomération de Montpellier et sa région en Languedoc, territoire à la fois pris d'assaut par les petites entreprises et les cadres et promu par ceux-là mômes qui s'y installent. 


\title{
Implantation de petites entreprises en zones aménagées
}

\section{Territoires à promouvoir ou territoires à prendre?}

\author{
Geneviève DUCHE \& Suzane SAVEY* \\ Université de Montpellier III
}

\begin{abstract}
RESUME
L'implantation et les modalités de fonctionnement (en particulier la nature des emplois offerts) de l'entreprise doivent s'inscrire dans un consensus minimum d'utilisation des hommes et de l'espace sans lequel il est difficile de créer les synergies nécessaires au développement local.

II paraít donc essentiel de comprendre les raisons de l'installation des entreprises dans un lieu plutót qu'un autre, $\infty$ qu'elles attendent de l'environnement local mais aussi ce qu'elles lui apportent, et les modalités de la rencontre entre leurs responsables et les b́lus locaux en charge de définir une politique de développement.
\end{abstract}

Pour tenter de répondre à ces questions, les auteurs ont travaillé sur quelques exemples de zones aménagées de l'agglomération de Montpellier et sa région en Languedoc, territoire à la fois pris d'assaut par les petites entreprises et les cadres et promu par coux-là mémes qui s'y installent.

\begin{abstract}
The location and style of management of firms (particularly the nature of jobs offered) needs a minimum degree of consensus on the use of manpower and space without which the synergies demanded by local development cannot be reached.

It is therefore of paramount importance to understand why firms settle in one location rather than another; to assess what they expect from the local environment, and also to appreciate what

- Les auteurs sont respectivement économiste et géographe. Elles se sont spécialisées dans le phénomène de spatialisation des P.M.E. en région. Elles ont publié des articles dans les revues d'économie régionale et de géographie. Leur adresse : Centre de recherche en économie sociale, Université Paul Valéry, Montpellier III, B.P. 5043, 34032 Montpellier Cedex, France. Première version reçue en décembre 1988.
\end{abstract}


they bring to it and how their executives meet the local elected representatives in charge of development policy.

The authors address these questions through a survey of several examples of developing areas in the city of Montpellier and the surrounding region, in Languedoc, a territory both literally conquered by small firms and their managerial staff and promoted by the very people who settle there.

\section{RESUMEN}

La implantanción y las modalides de funcionamiento (especialmente el tipo de empleos ofrecidos) de la impresa tienen que corresponder a un consenso mínimo de utilizacion de los hombres y del espacio sin el cual resulta dificil crear las sinergías necesarias para el desarrollo local.

A consecuencia, parece imprescindible entender las causas de la instalación de unas empresas en un lugar de preferencia a otro, lo que éstas pueden esperar de las circunstancias que las rodean y al mismo tiempo los beneficios que les pueden traer, los modalidades de encuentro entre sus ejecutivos y las autoridades electas del lugar encargadas de definir una politica de desarrollo.

Para intentar dar una respuesta a estas preguntas, los autores de este artículo han estudiado algunos ejemplos de zonas acondicionadas en conjunto urbano de Montpelliery en su periferia, en Languedoc, region de ésta, a la vez muy codiciada por las pequeñas empresas y los cuadros y promovida por los mismos que se instalan en ella. 
Le plus souvent, lorsqu'on aborde le thème du développement local, on est tenté d'orienter sa mesure, et parfois même exclusivement, en termes de croissance des emplois. La nécessité de lutter contre les effets réducteurs en effectifs salariés des changements structurels et organisationnels contemporains a enraciné l'idée que le développement équivaut surtout à l'augmentation des postes de travail en un lieu donné pour une période donnée.

De ce fait, aux vieilles références spatiales du local - villages, communes, cantons ou pays - s'est substituée celle du bassin d'emplois dont l'identification a pour objectif majeur de recenser les énergies pour mieux les mobiliser, pour gagner la bataille de l'emploi.

Dans ces circonstances particulières, l'entreprise, et tout particulièrement la petite entreprise, fait l'objet de toutes les attentions. Depuis longtemps, on sait que sa montée dans les années soixante-dix, son explosion dans les années quatre-vingts est seule capable, pour l'instant, de colmater tant bien que mal les brèches ouvertes par le délestage des effectifs des grandes entreprises.

Il peut donc paraître tentant de définir l'articulation développement local/ petites entreprises comme la part prise par les petites entreprises dans la croissance des effectifs salariés dans un bassin d'emploi donné.

Une telle définition ne nous satisfait pas.

D'une part elle est profondément réductrice par rapport à l'idée que nous nous faisons du développement et d'autre part elle évacue d'emblée la recherche de la dimension spatiale la mieux adaptée pour promouvoir le développement.

Sans proposer immédiatement une définition du développement local nous souhaitons insister d'abord sur les conditions nécessaires à son existence.

La première condition concerne la mise en œuvre d'une très forte capacité d' organisation des acteurs locaux qui leur permet de valoriser les ressources locales et/ou importées et de réinvestir sur place le produit de la valorisation de ces ressources. Le réinvestissementà partir du pôle émetteur localisé induit la croissance économique, par autocorrélation spatiale, del' environnement immédiat, et le maintien de cette croissance dans la durée, par autocorrélation temporelle. Il s'ensuit une croissance démographique par maintien sur place des populations qui auraient pu alimenter l'exode sans la mise en place de ces nouvelles formes d'organisation, mais aussi par l'arrivée d'une population de l'extérieur.

Toutefois l'arrivée de migrants n'est pas obligatoirement liée aux effets de la croissance économique qui se propage au niveau local. Les conditions générales du cadre de vie : environnement naturel, conditions climatiques, capacités d'accueil en logement, en infrastructures culturelles et de loisirs, peuvent également avoir des effets attractifs sans rapport immédiat avec la perspective de trouver tout de suite un emploi. L'augmentation de population n'est donc pas forcément un signe de la croissance économique, mais cette dernière induit le croît démographique. 
Néanmoins, la dynamique ainsi créée par la capacité des acteurs locaux à investir et à réinvestir sur place en vue de valoriser les ressources, et l'augmentation des hommes et des activités qu'elle entraîne conduisent à une complexification de l'organisation des activités et des rapports sociaux. Cette complexification comporte en elle-même des risques de germination de tendances entropiques contradictoires avec le développement local.

En d'autres termes, le développement local ne saurait s'épanouir sans un consensus minimum entre les différents partenaires de l'espace socio-économique local, sans une mobilisation en vue d'objectifs précis et cohérents.

La seconde condition du développement local reposerait en effet sur la conscience que les acteurs concernés peuvent avoir de former un groupe cohérent, uni pour des objectifs communs et surtout lié par l'appartenance à la même unité spatiale. Les cohésions des hommes entre eux et des hommes avec les lieux seraient les supports actifs du développement local. Il apparaît donc ainsi que la dimension spatiale du développement local n'est pas prédéterminée par un découpage administratif quelconque : il peut s'agir aussi bien d'un regroupement de communes dans le cadre de syndicats inter communaux, de chartes inter communales, etc... que de la commune elle-même. Tout dépend en fait de la nature du regroupement en hommes, de la capacité d'organisation dont ils font preuve et de la surface relationnelle des leaders. On est renvoyé alors à l'identification des personnalités marquantes capables d'enlever l'adhésion de ceux qui seront les acteurs principaux et les troupes du développement local capables de faire naître de la collectivité un projet cohérent et de se donner, avec elle et grâce à elle, les moyens de le réaliser.

Latroisième condition du développement local serait donc ainsi profondément antinomique de l'Assistance. Certes il ne s'agit pas pour les communes, regroupées ou non, de faire fi des subventions ou prêts que la Région ou l'État peuvent leur accorder. Il ne s'agit pas davantage pour les entreprises de ne pas bénéficier des aides prévues pour les circonstances locales dans lesquelles elles cherchent à s'insérer. Mais il s'agit pour les uns et les autres de construire un projet cohérent tenant réellement compte des ressources existantes en hommes, en produits, en cadre de vie, ainsi que du potentiel qu'elles représentent. Il ne peut rien sortir de l'attribution quelconque de subventions s'il n'existe pas aussi la volonté de les utiliser dans la logique du projet de vie collectif défini et accepté par le plus grand nombre possible des habitants concernés. C'est seulement lorsqu'il existe et qu'il a déjà fait l'objet d'un début de mise en œuvre que l'aide financière des pouvoirs publics de plus haut niveau peut servir à la réalisation du projet et au développement local.

En bref, la définition d'une politique du développement local serait la forme achevée de la capacité à s'organiser pour atteindre les objectifs définis en commun ou collectivement acceptés. Ceci passerait par l'imagination de la bonne image a créer et à mettre en valeur; en d'autres termes, par la recherche des moyens susceptibles de produire une nouvelle valorisation de l'espace, porteuse de développement. 
Toutefois, la construction d'une telle image n'est pas chose aisée. Elle nécessite souvent la remise en cause de l'histoire, des traditions et parfois même, de l'identité collective des groupes concernés. Aussi les résistances sont-elles souvent nombreuses à sa définition et surtout quand les initiateurs d'une telle démarche sont des nouveaux venus dans l'espace local. Les plus grandes chances de succès paraissent être assurées quand l'initiative des nouvelles formes d'organisation et du changement d'image provient d'acteurs enracinés dans le local, en ayant une connaissance intime, et capables de convaincre de la nécessité des transformations, dans le respect de l'identité des hommes et des lieux.

Mais, par ailleurs, le changement d'image peut avoir des effets pervers sérieusement perturbateurs pour l'objectif à atteindre. Montpellier technopole, centre de culture, de matière grise et de rénovations urbanistiques grandioses n'attire pas seulement les cadres supérieurs mais aussi les femmes seules en quête d'emplois, les marginaux de toute nature et les chômeurs au soleil. Le triste record du taux de chômage dont elle ne parvient pas à se débarrasser n'est-il pas lié à ce changement d'image ? Certes l'espace a pris une autre valeur, certes les embellissements sont un atout pour l'avenir mais les montpelliérains de souche se sentent étrangers dans leur ville, la tour du Triangle fait office de «miroir aux chômeurs» et malgré la formidable explosion de créations d'entreprises, surtout petites, le problème de l'emploi évoque celui du tonneau des Danaïdes.

Dans ce cadre général en effet, l'entreprise - petite ou grande - est l'un des partenaires et seulement l'un des partenaires, mais tout à fait essentiel. Si nous acceptons les termes des conditions dans lesquelles le développement local peut s'épanouir et que nous venons de décrire, l'entreprise en tant que telle doit être l'une des composantes de cette capacité d'organisation des acteurs locaux susceptible de valoriser les ressources locales et/ou importées, et de réinvestir sur place le produit de la valorisation de ces ressources. Par ailleurs, l'implantation et les modalités de fonctionnement (en particulier la nature des emplois offerts) et de développement de l'entreprise doivent s'inscrire dans un consensus minimum d'utilisation des hommes et de l'espace sans lequel il est difficile de créer les synergies nécessaires à la réalisation d'objectifs précis et cohérents.

Dans ces conditions, il paraît tout à fait essentiel d'essayer de comprendre les raisons qui font qu'à un moment donné de leur trajectoire les entreprises choisissent de s'installer dans un lieu plutôt qu'un autre, ce qu'elles attendent de l'environnement local mais aussi ce qu'elles lui apportent.

En d'autres termes, est-il possible de concilier le nécessaire profit à réaliser par l'entreprise avec la promotion d'un développement local répondant aux critères que nous venons d'énumérer? Dans quelles conditions les implantations d'entreprises, fermement articulées aux politiques locales, peuvent-elles constituer l'un des supports les plus solides du développement de la micro-région ? Dans quelles conditions, au contraire, se comportent-elles seulement comme des greffons temporaires ou pérennes qui usent des ressources locales sans en créer de nouvelles et dont l'apport se résume à quelques emplois pas toujours durables ? 
Pour essayer de répondre à ces questions nous avons travaillé sur quelques exemples de zones aménagées dans l'agglomération de Montpellier et de sa région. Nous avons tenté de mettre en relation les motivations d'implantation des entrepreneurs avec les politiques municipales pratiquées afin d'examiner s'il se dégage une logique dans d'éventuelles correspondances d'attitudes entre ces deux types majeurs de partenaires. Enfin nous avons voulu savoir si les correspondances repérées pouvaient avoir un lien quelconque avec différents niveaux d'un développement local toujours sous la menace d'effets pervers difficiles à contrôler et surtout à maîtriser.

\section{Motifs d'implantation des entreprises et politiques municipales}

Tenter de comprendre l'articulation entre les politiquesmunicipales, l'implantation des petites entreprises et le développement local dans une zone géographique tout entière dominée par une capitale régionale telle que Montpellier n'est pas une entreprise très facile. Comment faire la part de ce qui revient aux dynamiques purement locales et de ce qui ressort des retombées de la métropole ?

Comment démêler dans l'imbroglio politique héraultais ce qui correspond à des initiatives de communautés locales représentées par leurs élus de ce qui se réalise en fait parce que ces élus ou d'autres notables ont une surface politique ou simplement un entregent tels que leurs propositions peuvent devenir réalité, même si leurs administrés ne les poussent pas ni même ne les suivent?

L'ampleur de la tâche est telle que pour cette première approche nous avons choisi de simplifier la procédure en choisissant de travailler sur des zones aménagées (ZI,ZA,ZAE) dans Montpellier (Près d'Arènes, Zolad), dans sa périphérie immédiate (Le Crès, Vendargues à l'Est et Juvignac, St-Georges-d'Orques, St-Jean-de-Védas à l'Ouest) et sur trois pôles plus lointains situés à peu près à égale distance de la ville : St-Mathieu-de-Tréviers au Nord, Le Grau-du-Roi au Sud-Est, Clermont-l'Hérault et St-André-de-Sangonis à l'Ouest.

L'analyse repose sur une enquête réalisée en 1986 auprès de 191 entreprises de moins de 50 emplois (dont 105 de moins de 10), lesquelles représentent au minimum $25 \%$ des implantations et au mieux $60 \%$ environ. L'enquête a été complétée par des entretiens avec les élus municipaux et une autre enquête auprès des secrétaires de mairie.

Dans l'enquête menée auprès des chefs d'entreprise, nous avons essayé de savoir quels avaient été les motifs ayant guidé leur implantation sur la zone aménagée choisie. 
Pour cela nous avons retenu 14 critères pour lesquels les réponses fournies paraissent suffisamment significatives, compte tenu des non réponses :

- 4 critères qui relèvent de toute zone aménagée, quelle que soit sa localisation sur l'espace national. ( 1 : facilités d'accès, 2 : terrains disponibles équipés, 3 : prix des terrains, $4:$ superficie des parcelles)

- 7 critères qui concernent directement les caractéristiques du milieu local. (5 : présence d'un marché local, $6:$ présence de matière première locale y compris matière grise, 7 : proximité des banques, $8:$ solidarités techniques possibles, 9 : main-d'œuvre qualifiée disponible, 10 : environnement culturel offert par l'agglomération de Montpellier, 11 : qualités physiques de l'espace local).

- 3 critères qui relèvent de l'aide accordée par la puissance publique (12 : subvention d'installation, 13 : prime par emploi cré, 14 : allégements fiscaux).

Chaque chef d'entreprise a dû se prononcer sur chacun de ces critères et estimer si, pour son propre choix d'implantation, il avait été très important, important peu important ou sans importance.

En regroupant les réponses très important et important pour chacun des critères, la totalisation des réponses pour chacun des secteurs géographiques est exprimée dans le tableau 1 (p. 79) en valeur absolue et en pourcentage.

A partir de ce tableau, un classement a été effectué en calant les résultats obtenus par chacune des zones géographiques retenues par les résultats moyens de l'ensemble de l'échantillon. Les zones dont les résultats en pourcentage des réponses dépassaient le pourcentage moyen des réponses de l'échantillon global ont été considérées comme suffisamment attentives au critère envisagé et ont été dotées d'un point pour ce critère, dans le classement (tableau 2, p. 81).

Ce classement repose donc sur le degré d'attention portée aux différents motifs d'implantation proposés a priori par le questionnaire d'enquête aux chefs d'entreprise et sur la perception qu'ils ont, a posteriori, des motifs qui ont pu guider leur choix. Il repose aussi sur l'idée que lesrésultats moyens obtenus sontreprésentatifs de l'ensemble. Enfin, il faut encore tenir compte que tous les entrepreneurs sollicités n'ont pas consentià répondre à l'enquête et que ceux qui ont bien voulu répondre sont peut-être ceux qui ont effectivement le plus analysé au départ le contenu de leur prise de décision. On peut saisir ici toutes les limites de la méthode utilisée. Néanmoins, comme l'ensemble de ces défauts se répartit également sur tous les secteurs géographiques étudiés, on peut tout de même essayer de proposer prudemment quelques interprétations.

Il est en effet remarquable que les entrepreneurs des différentes zones d'enquêtes ne se comportent pas de la même façon dans les réponses qu'ils donnent à la question du choix de leur implantation. Dans certaines zones comme St-Mathieude-Tréviers, le Grau-de-Roi ou le Crès, les entrepreneurs paraissent avoir manifestement plus réfléchi qu'ailleurs aux avantages offerts par la localisation choisie. A 
Clermont-l'Hérault St-André-de-Sangonis et à Juvignac St-Georges, la prise en compte des divers critères proposés, et en particulier ceux qui relèvent du potentiel local, est beaucoup plus rare. Quant aux quatre zones dont l'organisation relève du District de Montpellier, elles occupent une place intermédiaire qui pourrait exprimer que d'une certaine manière, en s'implantant à Vendargues, aux Prés d'Arènes, à la Lauze ou à la Zolad, les entrepreneurs ont partiellement fait confiance au District.

Mais on peut aussi penser que les différences d'attitudes observées proviennent de disparités liées aux caractéristiques des entreprises: date d'installation, taille ou nature de leur activité.

En effet,si l'on considère l'ensemble de l'échantillon on observe que la prise en considération de certains des critères de choix proposés par l'enquête est modulée par chacune de ces trois caractéristiques.

- Plus la date d'installation est récente, plus on accorde de l'importance à certains avantages présentés par les ressources locales (marché local, présence de matière première y compris matière grise, proximité des banques, solidarités techniques) et à l'aide de la puissance publique (subvention d'installation, prime par emploi créé, allégements fiscaux).

- Plus l'entreprise est de petite taille, plus l'entrepreneur semble accorder de l'importance aux ressources locales (marché local, banques, solidarités techniques, main-d'œuvre qualifiée, environnement culturel, cadre physique) et à l'aide de la puissance publique (subvention, prime à l'emploi, allégements fiscaux).

- Par ailleurs, les petites entreprises de l'industrie paraissent également accorder plus d'importance que celles des services aux ressources locales (matières premières, banques, solidarités techniques, main-d'œuvre qualifiée, environnement culturel, cadre physique) mais aussi aux prix des terrains et à l'aide de la puissance publique (subvention, prime à l'emploi, allégements fiscaux.

Autrement dit, les conditions de réflexion optimales quant à l'implantation et en particulier la prise en compte des ressources locales intrinsèques ou attribuées par le niveau national de l'aménagement du territoire paraissent être réunies dans l'entreprise industrielle de moins de 10 emplois et d'implantation récente, pour l'échantillon considéré.

Le classement des différentes zones géographiques que nous avons effectué s'appuie sur d'autres facteurs que les caractéristiques des entreprises.

Il s'agit des dynamismes locaux engendrés par les synergies créées par l'entente des différents partenaires du développement local.

Si nous prenons en compte l'histoire des différentes zones et les liens qui ont pu se créer entre les chefs d'entreprise et les représentants du pouvoir local, trois groupes se dessinent : 
Tab. 1.

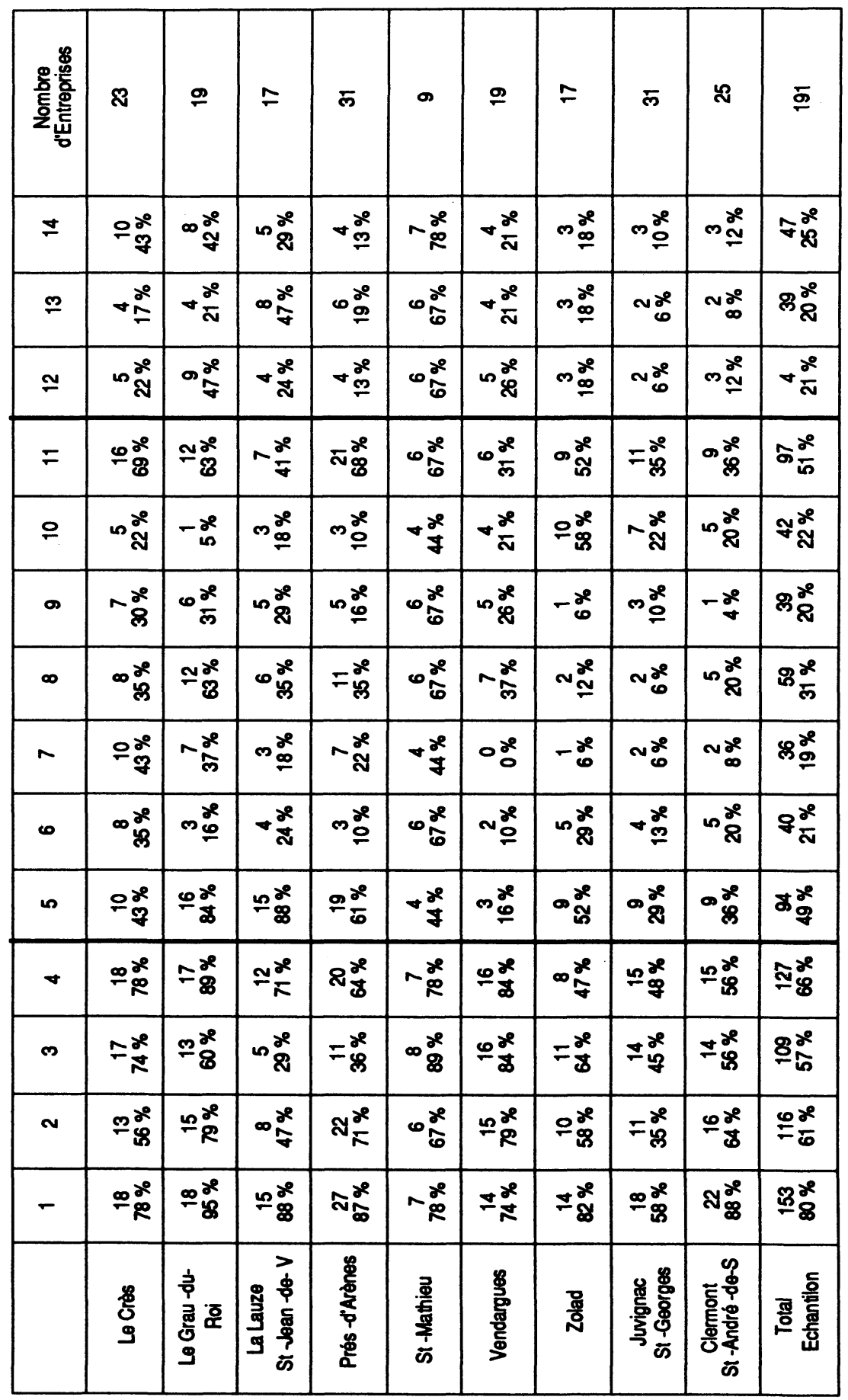


- celui des initiatives locales précoces facilitées depuis plus de 15 ans par une entente active (St Mathieu, Le Grau) ou passive (Le Crès) de la part des édiles entre les entrepreneurs et les Municipalités.

- celui des initiatives contrôlées ou imposées par un organisme de niveau supérieur, le District, qui peut dans certains cas faire figure de puissance extérieure au niveau local, même si sa compétence n'est pas contestée, (St-Jean, Vendargues, Prés-d'Arènes, Zolad) dans la mesure où ses options visent à l'organisation de la capitale régionale et non à celle du développement des différentes communes en tant que telles.

- celui des initiatives locales tardives de liaisons municipalités-entreprises dans la mesure où les préoccupations viticoles sont restées très longtemps dominantes pour les élus locaux (Clermont/St-André, Juvignac/St Georges).

Les initiatives locales précoces réalisées dans les trois communes têtes de file de notre échantillon révèlent une capacité d'organisation importante reposant sur un consensus entre les chefs d'entreprise et les élus locaux.

\subsection{Un exemple : St Mathleu : satisfaire les Industriels et pratiquer une politique d'accompagnement}

A la fin des années soixante, ce qui deviendra la zone à usage industriel, artisanal et commercial de St-Mathieu-de-Tréviers se crée un peu par hasard : par le déménagement d'une petite entreprise sous-traitante d'IBM, employant cinqpersonnes et jusque là installée dans un hangar agricole. L'extension de l'entreprise qui diversifie ses activités pour se libérer partiellement de sa dépendance vis à vis d'IBM, qui accroît ses effectifs au-delà de 100 emplois et qui par ailleurs intéresse la populationà son développement $(1 / 3$ deson financement estassuré par lescommerçants du village) satisfait la municipalité mais l'inquiète également. Très certainement choquée par l'exemple de Ventex dont l'implantation fugace a précipité le déclin de la région gangeoise, la Municipalité de St-Mathieu réfléchit aux conséquences aléatoires pour le développement local de l'installation en solitaire d'une entreprise de grande taille relative, maîtresse de l'emploi d'une commune ou d'un canton et dispensatrice de catastrophes en période de difficultés. Aussi, dès 1975, la municipalité se préoccupe-t-elle de multiplier et de diversifier les entreprises sur sa commune.

Deux axes sont choisis : satisfaire au mieux les industriels en mettant à leur disposition des terrains à bas prix (à 35F/le $\mathrm{m}^{2}$ viabilisé, les prix de St-Mathieu-deTréviers sont encore en 1986 les plus bas de la région), en garantissantà chacun d'eux la disponibilité pendant 3 ans de la parcelle qui jouxte leur établissement pour permettre les agrandissements éventuels, en les exonérant de la taxe professionnelle pendant les cinq années qui suivent leur installation, en mettant à leur disposition des ateliers relais qu'ils peuvent s'approprier à terme par location vente; pratiquer une politique d'accompagnement du développement des emplois par construction de 


\begin{tabular}{|c|c|c|c|c|c|c|c|c|c|c|c|c|c|c|c|c|}
\hline & 1 & 2 & 3 & 4 & 5 & 6 & 7 & 8 & 9 & 10 & 11 & 12 & 13 & 14 & $\begin{array}{l}\text { Total } \\
1 \text { à } 14\end{array}$ & $\begin{array}{l}\text { Total } \\
5 \text { à } 11\end{array}$ \\
\hline St - Mathiou & & $x$ & $x$ & $x$ & & $x$ & $x$ & $x$ & $x$ & $x$ & $x$ & $x$ & $x$ & $x$ & 12 & 6 \\
\hline $\begin{array}{c}\text { Le Grau -du- } \\
\text { Roi }\end{array}$ & $x$ & $x$ & $x$ & $x$ & $x$ & & $x$ & $x$ & $x$ & & $x$ & $x$ & $x$ & $x$ & 12 & 5 \\
\hline Le Crès & & & $x$ & $x$ & & $x$ & $x$ & $x$ & $x$ & & $x$ & $x$ & & $x$ & 9 & 5 \\
\hline $\begin{array}{l}\text { St Jean -de } \\
\text { Védas }\end{array}$ & $x$ & & & $x$ & $x$ & $x$ & & $x$ & $x$ & & & $x$ & $x$ & $x$ & 9 & 4 \\
\hline Vendargues & & $x$ & $x$ & $x$ & & & & $x$ & $x$ & & & $x$ & $x$ & & 7 & 2 \\
\hline Prés -d'Arènes & $x$ & $x$ & & & $x$ & & $x$ & $x$ & & & $x$ & & & & 6 & 4 \\
\hline Zolad & $x$ & & $x$ & & $x$ & $x$ & & & & $x$ & $x$ & & & & 6 & 4 \\
\hline $\begin{array}{c}\text { Clermont } \\
\text { St -André -de-S }\end{array}$ & $x$ & $x$ & & & & & & & & & & & & & 2 & 0 \\
\hline $\begin{array}{l}\text { Juvignac } \\
\text { St -Georges }\end{array}$ & & & & & & & & & & & & & & & 0 & 0 \\
\hline
\end{tabular}


logements sociaux, et mise en place ou extension d'infrastructures routières, commerciales, éducatives, culturelles et de loisirs.

En contrepartie, les entrepreneurs doivent accepter quelques contraintes, créer au moins 11 emplois en trois ans et faire un minimum d'investissements. D'autre part les transferts d'établissement ne sont pas acceptés, il doit s'agir de création.

Avec cette politique, la zone s'est progressivement peuplée de 14 entreprises qui représentent 450 emplois environ.

\subsection{Les Initlatives sous contróle : Montpellier et son District}

Parmi les zones étudiées, celles de Prés-d'Arènes, de laZolad, de Vendargues et de la Lauze représentent en réalité quatre pôles mis en place pour réorganiser l'industrialisation de l'agglomération montpelliéraine. Seule l'une d'entre elles, Prés-d'Arènes, est tout entière localisée sur la commune capitale, les trois autres sont installées en périphérie, partiellement sur Montpellier et Grabels pour la Zolad, en totalité sur St-Jean-de-Védas pour la Lauze et à une dizaine de kilomètres, à Vendargues, pour la dernière.

En fait, dès la fin des années cinquante, la réflexion municipale exprime le désir d'attirer à Montpellier les industries qui lui manquent et la création des Présd'Arènes en 1959 et du Marché Gare en 1962 vont dans ce sens. Bénéficiant des facilités offertes par l'État dans le cadre de l'Aménagement du Territoire, les deux opérations visent à créer des emplois à partir d'entreprises de conditionnement de produits agricoles, d'emballages, de conserveries mais aussi de fabricants d'engrais et autres compléments nécessaires à l'agriculture. L'idée avancée alors est de tirer parti de la reconversion agricole lancée par la CNABRL, d'essayer de fixer la population active libérée par l'abandon des terres viticoles exacerbé par la crise de mévente du vin, d'offrir un point de chute aux entreprises rapatriées d'Algérie et de diminuer le nombre des demandeurs d'emplois, 1500 environ au début des années 60.

Aussi, dans un premier temps, Montpellier s'occupe exclusivement de son territoire et selon le cahier des charges «les lots des Prés-d'Arènes sont destinés en priorité à des industries nouvelles dont la création aura pour effet d'offrir de nouveaux emplois [...] sont interdits sur la zone toutes les constructions à usage d'habitation ainsi que les constructions affectées à une activité commerciale de détail.» Mais la zone sert surtout en fait au décongestionnement du centre et au regroupement d'entreprises locales déjà existantes : sur les 52 parcelles vendues, 34 ont été achetées par des entreprises locales, 6 par des rapatriés et 12 par des entreprises extérieures. La majorité des emplois fixés sur la zone dans un premier temps a surtout concerné les services de transport, de réparation et de distribution, plus que la production proprement dite.

Mais malgré cela, la zone devient vite insuffisante pour répondre à la demande et, le projet d'une troisième tranche devant être abondonné en raison 
semble-t-il du coût trop élevé de l'expropriation nécessaire, d'autres localisations s'imposent. C'est à ce niveau qu' intervient le District. Créé en janvier 1965, il réunit alors 11 communes en plus de Montpellier, dont St-Jean-de-Védas, Grabels et Vendargues, et prévoit que dans ses attributions facultatives il peut être envisagé l'aménagement de zones industrielles par délibération du Conseil de District et accord des Conseils municipaux concernés.

Aussi, le 18 novembre 1965, un emprunt à la Caisse des Dépôts et Consignations est garanti par le District pour équiper la zone industrielle de Vendargues, à réaliser en grande partie sur des terrains communaux, et devant accueillir en priorité l'industrie lourde et semi-lourde que la ville de Montpellier ne pouvait pas intégrer. En 1973, une seconde tranche d'équipement est votée par le district et sa réalisation, comme celle de la première tranche, est confiée à la SERM un peu plus tard. Actuellement, une zone d'activités est en préparation, qui sera œuvre communale cette fois-ci, mais la municipalité compte encore sur le District pour la remplir.

Dans la même délibération du 18 novembre 1965, le district décide de créer la zone industrielle de la Lauze, sise à St-Jean-de-Védas, pour décongestionner, à l'Ouest, la zone industrielle des Prés-d'Arènes. L'aménagement est confié à la SERM. Depuis, 98 entreprises se sont installées permettant ainsi le rassemblement de près de 1000 emplois. Un peu plus tard, en 1968, l'implantation des laboratoires pharmaceutiques Bristol et Chauvin Blache invite la Municipalité de Montpellier à projeter une zone d'activités non polluantes qui déborde les limites communales pour s'étendre aussi sur Grabels, autre commune du district. Une fois encore, la responsabilité de l'opération revient au District. Les entreprises s'installent assez rapidement. Sur les 75 ha prévus, seuls 2 ou 3 restent actuellement disponibles.

D'un certain point de vue, on peut dire que toutes ces opérations ont réussi et que le District a bien rempli son rôle de réorganisation des implantations industrielles de l'agglomération, mais qu'advient-il dans ce cadre de l'initiative locale ? Certes les communes ont apporté dès le départ leur consentement à ces divers aménagements. Certes elles pouvaient espérer financer en partie les équipements nécessaires à l'augmentation de population qu'elles ne devaient pas manquer de connaître, même sans zone industrielle, avec la taxe professionnelle. Mais la grande partie de la taxe revient au District et la population communale ne cesse de croître. Vendargues, par exemple, est passée de 1869 habitants en 1975 à 2600 en 1982, 3578 en 86 et pense en compter 4000 fin 88 . Bien entendu, l'appartenance au District a ses avantages et le développement des transports en commun urbains en fait foi. Mais en vertu de son rôle d'aménageur de niveau supérieur, le même District a dépossédé les communes de leur capacité à envisager des projets autonomes. Les entreprises font figure de corpsétrangers même si leurs rapports avec les municipalités sont bons. Pas un seul chef d'entreprise dans le Conseil municipal de Vendargues. On a établi un bon modus vivendi mais les projets des entreprises ne sont pas ceux de la municipalité et réciproquement.

Les communes ont bien senti d'ailleurscette limitation d'initiative qu'entraîne leur participation au District. Il suffit pour s'en convaincre de lire l'histoire de leurs 
efforts pour faire tomber la participation des délégués de la ville de Montpellier en dessous du seuil des $50 \%$ et les efforts non moins grands du président du District pour rétablir la parité. Au-delà des luttes des tendances politiques, réelles, et qu'il ne faut pas sous estimer, existe aussi la volonté de reconquérir la capacité à décider de manière autonome. Même si les choix qu'elles pourraient faire ne prennent pas tout à fait en compte les impératifs généraux liés à la croissance d'une agglomération dont elles font partie, qu'elles le veuillent ou non, les communes du District, ou tout au moins certaines d'entre elles, aspirent à retrouver un certain statut d'adulte.

\subsection{Les Inltiatives locales tardives : l'Industrie de l'après-viticulture}

A Juvignac, St-Georges-d'Orques, St-André-de-Sangonis et Clermontl'Hérault, l'industrialisation n'est pas prise en compte, jusqu'à récemment, comme un facteur premier du développement local.

Un exemple à quelques kilomètres de Montpellier. Juvignac et St Georges sont encore, dans les années soixante, des villages types de sa «banlieue viticole». Avec sa cave coopérative et un millier d'habitants environ, St Georges fait figure de gros bourg languedocien traditionnel dont l'activité économique repose essentiellement sur un vin de qualité qui a reçu depuis 1944 le statut de VDQS. Beaucoup plus modeste, Juvignac qui n'atteint pas encore 200 habitants en 1962, vit de ses vignes, coincé entre Montpellier, qui déjà s'agrandit et se retrouvera bientôt à ses portes par absorption de Celleneuve, alors que St-Georges est beaucoup mieux doté en histoire, en habitants, et en potentiel viticole commercialisable.

La création de la ZUP de la Paillade au milieu des années soixante, aux limites de la commune traversée par l'axe Montpellier-Lodève, et la présence de terrains viticoles relativement peu chers en bordure de cet axe ont, comme au Crès, attiré des implantations spontanées d'entreprises. A l'écart des zones habitées, les vignes vendues aux entrepreneurs permettaient l'installation, sans problème de nuisance, d'activités éventuellement polluantes du point de vue du bruit ou de la poussière. Aussi, une zone industrielle de fait sans statut et sans contrôle se développe-t-elle très librement jusqu'en 1971 où le premier POS reconnaîtra le fait accompli alors que l'autre côté de la route Montpellier-Lodève verra se multiplier les lotissements du Plan Chalandon. Les entreprises de travaux publics, de production de béton, de maçonnerie et autres, en tout une soixantaine, se trouvent désormais insérées en pleine ville à proximité des habitations qui rassemblent maintenant près de 4000 habitants.

De ce fait, malgré l'intérêt, évident pour la commune, de se nourrir de la taxe professionnelle, l'objectif n'est pas de créer des emplois à partir d'une extension ou d'une création nouvelle de zone industrielle, artisanale ou d'activités. La nouvelle image à créer passe par tout autre chose. Pour la municipalité actuelle l'extension des activitésà Juvignac doit reposer sur l'exploitation du potentiel loisir et le thermalisme. 
En achetant les 180 ha du domaine de Fontcaude, la municipalité veut faire renaître l'utilisation d'une source chaude qui fut exploitée dans l'Antiquité romaine, au Moyen Age et au siècle dernier. Un golf, des hôtels, un Club House, une zone d'habitations de 35 ha, une salle de spectacles et de rencontres devraient assurer 200 à 250 emplois dans les cinq années à venir. Dans ces projets, et malgré la négociation en cours de l'achat de 58 ha pour le commerce et l'artisanat, il apparaît que l'activité productive de l'industrie est plus perçue comme un handicap que comme un atout, en raison des modalités actuelles de sa localisation que l'on souhaiterait transférer.

A St-Georges, l'histoire de la zone industrielle aurait pu être très semblable de celle de Juvignac si la structuration ancienne du village n'avait imposé d'ellemême une localisation périphérique. Là aussi, tout commence par des initiatives spontanées: 1'installation de l'entreprise de travaux publics des frères BEC, en 1972, puis de Technic Offset vont déterminer la localisation de la zone d'activités en bordure de la nationale 109. Lancée par la municipalité, l'opération offre 23 parcelles dans la première tranche, 24 beaucoup plus petites dans la seconde, les plus grandes parcelles de la première tranche ayant trouvé difficilement acquéreur. Certes peu à peu les choses s'organisent et la liaison Municipalité-entreprise se construit progressivement. Des chefs d'entreprise entrent au conseil municipal, Technic Offset collabore à la réalisation de la plaquette publicitaire de la zone, on réfléchit ensemble à la meilleure manière d'améliorer l'image de la deuxième tranche par rapport à celle de la première, mais la préoccupation première de la commune reste le vignoble, la réputation du vin, l'amélioration de sa qualité et le fonctionnement de la cave coopérative. Pas étonnant que dans ces conditions, les habitants de St-Georges ne se sentent pas ou peu concernés par une zone artisanale qui certes a créé des emplois mais pas obligatoirement pour des résidents de St-Georges et qu'en plus, ils ne fréquentent guère car elle se trouve très à l'écart de leurs trajets quotidiens.

Ainsi les hypothèses que l'on peut provisoirement formuler à partir de l'analyse de ces quelques communes seraient les suivantes :

- l'attention portée au milieu local par les entrepreneurs est d'autant plus grande que la capacité collective d'organisation est ancienne, qu'elle provienne des municipalités (St-Mathieu-de-Tréviers) ou des entreprises elles-mêmes (Le Grau-du-Roi, le Crès).

- l'attention portée au milieu local par les entrepreneurs est d'autant plus grande qu'ils ont trouvé depuis longtemps dans les municipalités des interlocuteurs compréhensifs face à leurs problèmes mais suffisamment fermes pour éviter des localisations rejetées ensuite par les populations. L'absence de fermeté dans le passé expliquerait peut-être la situation actuelle de Juvignac et la priorité accordée, dans le passé également, à la viticulture plutôt qu'à l'industrie et aux services, la situation de St-Georges, Clermont et St-André.

- l'attention portée au milieu local par les entrepreneurs est d'autant plus grande que le niveau spatial d'intervention est restreint, ce qui expliquerait l'intérêt assez faible accordé à l'environnement local des entrepreneurs installés sur les zones aménagées par le District. 
Toutefois, il semble clair enfin que la notion de niveau local est imprécise et en pleine évolution. Pour le maire de Montpellier, président du District, la dimension spatiale du local est de toute évidence celle d'un district si possible de plus en plus en vaste. Pour le maire de St-Mathieu-de-Tréviers, président du Conseil Général de l'Hérault, la dimension spatiale du local est celle d'une association des communes de la zone nord de Montpellier, quelle que soit le nom qu'elle porte, ceci pour se protéger des empiétements à craindre par l'extension de la capitale. Pour le sénateur maire de Clermont-l'Hérault, Conseiller Général, la dimension spatiale du local se dessine dans l'avenir par le regroupement des quatre villes proches de la moyenne vallée de l'Hérault (Gignac, Lodève, Pézenas, Clermont). Pour le maire du Crès, la dimension spatiale du local réside dans l'association de communes rurales, pas toujours les mêmes, en vue de la réalisation d'objectifs communs et ponctuels et de manière à échapper au contrôle du District. Enfin, pour d'autres, la dimension spatiale du local reste encore dans les frontières de la commune à l'intérieur desquelles on recherche une spécificité propre à attirer les hommes et les activités.

Dans tous les cas, l'idée générale qui se dégage est qu'il n'est de richesse que d'hommes et le projet d'une limitation de leur croissance en nombre n'apparaît pas encore, ou de manière très ténue.

Est-il possible, dans ces conditions, d'assurer un développement à la mesure de la croissance, d'attendre autre chose des entreprises que d'être des pourvoyeuses d'emplois?

\section{Territoire pris et promu}

Le territoire au sens d' «espace-sol» est l'élément le plus passif du développement économique. Il peut contenir des richesses exploitables, être traversé par des voies de communication naturelles ou offrir un climat favorable à la vie humaine, et ainsi contenir des potentialités pour la croissance économique.

Mais ces atouts éventuels ne sont transformables en facteurs actifs de production que si le territoire ou certains de ses aspects sont «agis» par l'homme. Il est nécessaire que les conditions de développement scientifique, technique et culturel de la société en rapport avec ce territoire permettent de tirer profit de tout ou partie de ses caractéristiques.

Il est courant de rencontrer dans l'histoire du monde des régions non développées à sous-sol regorgeant de richesses, des zones de climat rigoureux économiquement très actives. Le territoire sera donc toujours à prendre et les créateurs de richesses matérielles choisiront tel territoire selon les atouts qu'il offre en relation avec le mode d'accumulation et la nature des secteurs moteurs. La richesse du sous-sol en minerai ne fait plus le dynamisme de certaines régions minières; la 
nécessité de la grande vitesse fait délaisser les voies d'eau naturelles; les nouveaux moyens de communication permettent délocalisation et décentralisation des firmes... Les critères de choix des territoires à investir pour l'installation d'entreprises évoluent avec le développement économique et ses déterminants.

Le Languedoc, terre des vignes et des garrigues, semblait ne jamais devoir intéresser et attirer les industriels et les créateurs d'entreprises. Ce territoire n'était pas un «espace-sol» de développement industriel. Des construits sociaux s'y étaient développés hors des sentiers de la modernité d'hier. Tout au plus, ses caractéristiques géographiques pouvaient en faire un lieu de tourisme et de loisir.

Brusquement, avec les mutations socio-économiques, ce qui faisait handicap s'est transformé en atout et le Languedoc, en même temps qu'un certain nombre d'autres régions françaises, s'est trouvé promu dans le basculement de la «France Inverse» (Uhrich, 1987). Cette périphérie devenait territoire à part entière, à prendre pour une installation durable et plus seulement pour le temps d'une escapade au soleil, d'une aventure dans les Cévennes, ou de congés payés sur le front de la plage.

La rapidité du changement ne doit par faire oublier les efforts d'aménagement du territoire qui avaient tenté de corriger les inégalités et de combattre la désertification. Les politiques d'aménagement ont eu des effets en Languedoc-Roussillon et ont permis l'implantation de quelques établissements de grandes firmes. De leur côté, les municipalités des grandes villes n'ont pas attendu la crise pour équiper des zones industrielles et tenter d'attirer de nouvelles entreprises.

Il s'agissait avec ces aménagements d'homogénéiser le territoire national, c'est-à-dire d'apporter les infrastructures et les équipements nécessaires en tout lieu propice à l'implantation et au développement des entreprises. Les caractéristiques naturelles ou la proximité des centres très actifs pouvaient passer au second plan puisqu'on pouvait développer partout les transports routiers (autoroutes) et aériens (aéroports) et la circulation des informations (téléphone, télex...). L'électricité et le béton irriguant le territoire, il suffisait d'ajouter des aides à l'implantation sous forme de subventions à la création d'emploi, de dégrèvements fiscaux, pour attirer les entrepreneurs. Les plus persuasifs pouvaient l'emporter.

Mais il a fallu attendre que les conditions d'accumulation changent pour que ces infrastructures portent leurs fruits et que le cadre général mis en place (le District de Montpellier date de 1965) serve de base à un développement rapide des implantations d'entreprises (tableau 3, p. 89 - le trait vertical épais marque l'accélération des créations d'entreprise dans les communes étudiées).

Des caractéristiques spécifiques du Languedoc devenaient alors les leviers du foisonnement des petites entreprises et de l'arrivée massive de nouveaux languedociens. Il s'agissait alors, pour un certain nombre d'acteurs, de prendre un territoire «espace-construit» qui porterait les clés d'un nouvel ordre productif. (Par «espace-construit» on entend un espace façonné par l'histoire et donc par l'action des individus et des groupes.) 
Implantation de petites entreprises en zones aménagées

Le Languedoc offre une main-d'œuvre peu qualifiée, prête à des salaires modestes et sans tradition syndicale parce que le territoire a peu connu l'industrialisation. Par ailleurs, ce même territoire contient les ingrédients d'un cadre de vie et de modernité à la californienne, avec des qualités physiques de l'environnement et l'existence d'une matière grise importante mise en œuvre dans les centres de recherche et l'Université.

Dans ce contexte, les entrepreneurs interrogés ne peuvent que privilégier dans leurs motifs d'implantation ce qui est spécifique à la région ou ce qui est relativement rare (les zones aménagées n'étant plus rares) en France. Plus leur implantation est récente, plus ils focalisent leurs réponses sur les caractéristiques du milieu local, juste récompense des responsables politiques qui depuis trois, quatre ans mettent en valeur ces spécificités devenues éléments moteurs du développement économique.

Tout se passe comme si un gisement caché avait été découvert et exploité avec l'assentiment et la ferveur de tous. Un retour au tableau 3 (p. 89) montre que dans la majorité des communes étudiées $50 \%$ des entreprises existantes ont été créées seulement à partir de 1984 et 1985.

Territoire à prendre ou à promouvoir? La réponse à cette question apportera un nouveau paradoxe à cette région laquelle connaît déjà la coexistence d'un chômage très élevé et d'un taux important de création d'emplois.

Son territoire est à la fois pris et promu... Pris d'assaut par les créateurs d'entreprises qui en majorité ne sont par originaires de la région, pris par ces nouveaux habitants plutôt jeunes et, dans l'ensemble, qualifiés (cadres) qui mettent en cuvre la mobilité attendue d'eux dans la nouvelle culture d'entreprise et qui entraînent avec eux les exclus et les nouveaux pauvres attirés par l'image d'une région où tous les espoirs semblent permis, où, en tout cas, on pourra tendre la main dans un climat plus clément.

Promu parce que ceux-là même qui s'y installent en font la promotion en lui reconnaissant des atouts et en lui apportant ce qui lui manque. Mais surtout, promu parce que, et c'est là que résiderait le paradoxe, les / «envahisseurs» ou les pionniers ont souvent été bien accueillis. Au-delà d'un territoire «espace-construit» / terre à prendre, ils ont trouvé un territoire «espace-projet» façonné par des responsables locaux conquis par la nouvelle modernité. Depuis longtemps, la plupart des responsables politiques (maires) avaient pris conscience de la nécessité des restructurations économiques (problèmes des zones rurales) et de l'émergence d'une mentalité entrepreneuriale pour un développement local. Leur volonté d'intervention était - et est toujours - axée sur la création d'emplois. Mais peu d'entre eux avaient la volonté ou les moyens de mettre en place un véritable développement local endogène. (Soulage, 1987)

Dans leur recherche d'opportunités de gains, les néo-languedociens apportent des savoir-faire et savoir-être, les «locaux» apprennent de nouveaux comportements 
Tab. 3. Distribution des entreprises existantes en 1987 selon leur date de création, en \%

\begin{tabular}{|c|c|c|c|c|c|c|c|c|c|}
\hline & $\begin{array}{c}\text { Avant } \\
80\end{array}$ & 80 & 81 & 82 & 83 & 84 & 85 & 86 & 87 \\
\hline $\begin{array}{l}\text { St-Georges- } \\
\text { d'Orques }\end{array}$ & 26,6 & 0 & 5 & 8,3 & 5 & 6,6 & 21,6 & 15 & 10 \\
\hline Juvignac & 22,3 & 7,1 & 4,0 & 4,0 & 4,0 & 17,1 & 7,1 & 19,1 & 14,1 \\
\hline Le Crés & 21 & 6,1 & 4,3 & 3,7 & 3,7 & 13,6 & 8 & 17,9 & 21,6 \\
\hline Vendargues & 25,8 & 2,8 & 3,4 & 4,5 & 7,5 & 6,3 & 12,06 & 24,1 & 10,9 \\
\hline $\begin{array}{l}\text { StJean- } \\
\text { de-Védas }\end{array}$ & 25,3 & 0,7 & 2,9 & 4,7 & 2,7 & 8,7 & 12,7 & 26 & 16,3 \\
\hline $\begin{array}{l}\text { Clemmont- } \\
\text { I'Hérault }\end{array}$ & 40,8 & 3,2 & 1,7 & 5,7 & 3,3 & 5 & 11,8 & 12,9 & 14,3 \\
\hline $\begin{array}{l}\text { St-André } \\
\text { de-Sangonis }\end{array}$ & 40,2 & 5,2 & 2,6 & 3,9 & 2,6 & 9 & $\overline{11,7}$ & 14,3 & 10,4 \\
\hline $\begin{array}{l}\text { St-Mathieu- } \\
\text { de-Tréviers }\end{array}$ & 18 & 4,3 & 2,8 & 4,3 & 17 & 4,3 & 16 & 14,3 & 20 \\
\hline $\begin{array}{l}\text { Le Grau- } \\
\text { du-Roi }\end{array}$ & 33,9 & 3,9 & 6,2 & 6,8 & 6,7 & 8,3 & 11 & 10,2 & 12,5 \\
\hline
\end{tabular}

Fait à partir des sources $\mathrm{CCl}$ Montpellier 
Implantation de petites entreprises en zones aménagées

et sont souvent les garants du consensus nécessaire à l'œuvre de développement endogène basé essentiellement sur les ressources humaines. Dans la majorité des communes que nous étudions ici (hors capitale régionale), les maires sont originaires du département et le plus souvent de la commune qu'ils administrent.

Il apparaît ainsi dans cette rencontre entre entrepreneurs et territoire que la personnalité des élus locaux est un élément important pour le choix d'implantation. (Cognée et Vernazobres, 1985)

La décentralisation a renforcé le pouvoir et le rôle des élus. L'exemple de l'Hérault montre à quel point les entrepreneurs sont eux-mêmes des «territoires» que se disputent des élus compétents et montre comment des hommes politiques acharnés (Maire de la capitale et Président du Conseil Général surtout) peuvent passionner la population à propos d'un débat économique en doublant le marché concurrentiel des marchandises par un marché politique des idées, des images et des innovations.

La transformation du territoire languedocien en une région motrice de l'Europe se heurte cependant aux pesanteurs des habitudes, au retard des zones rurales creusé par la course à la modernité, aux clivages politiques et aux querelles de clochers.

Il est difficile de généraliser à partir des quelques zones observées, mais il apparaît très rapidement que le découpage administratif n'est pas forcément le cadre le plus adapté aux initiatives locales; l'étroitesse du cadre communal empêche une promotion autonome du territoire. Il dépend des acteurs locaux et des élus de favoriser et de construire les regroupements efficaces en termes de développement.

Le groupement qui semble porteur du dynamisme le plus important est celui du District qui a été relancé autour de Montpellier atteignant la masse critique nécessaire à une politique d'entraînement par polarisation d'activités clés pour la modemisation.

Une capitale rayonnante se construit. Elle structure l'espace environnant et ajoute au partagezone rurale/zone urbaine le partage appartenance ou non appartenance au District.

Quatre des communes choisies font partie du District : Juvignac, le Crès, Vendargues, St-Jean-de-Védas; les distributions des entreprises selon leur date de création y sont semblables (tableau 3), ce qui indique un développement des activités économiques récent et lié à la dynamique du District. St-Georges-d'Orques, hors District mais géographiquement très proche de Juvignac, semble suivre le même rythme d'implantation d'entreprises.

On a affaire au départ à de petites communes vivant dans l'orbite de Montpellier qui ont connu d'abórd un essor de leur population et une urbanisation explosive puis un croît plus récent des entreprises et commerces offrant des emplois sur place.

A cette ressemblance (communes de l'agglomération et d'abord villes dortoirs), s'ajoute une structure économique voisine. Le taux des industries y est 
relativement élevé, les activités du bâtiment y prennent une place encore plus importante (les activités du bâtiment regroupent industries des matériaux, bâtiment proprement dit et commerces de matériaux).

Ce groupe du District pourrait s'opposer au groupe formé par le Grau-duRoi, Clermont-L'Hérault, St-André-de-Sangonis et St-Mathieu, constitué de communes rurales. Cependant la commune de St-Mathieu-de-Tréviers présente une structure économique proche de celle des communes du District et une croissance récente des implantations d'entreprises.

Les trois autres se caractérisent par un poids très important des entreprises de service et un taux élevé d'entreprises créées avant 1980. Le Grau-du-Roi se distingue par une économie touristique qui se développe régulièrement depuis 1980, alors que Clermont-L'Hérault, à l'intérieur des terres, tente un démarrage difficile et reste le bourg qui offre à la population environnante une activité de commerce de détail traditionnelle.

D'apparentes ressemblances cachent des différences de processus et de politiques de développement. A l'intérieur du district, la situation sur les axes de communication donne les mêmes atouts à St-Jean-de-Védas, le Crès et Vendargues, mais les voies prises pour le développement n'y sont pas les mêmes.

Les communes de Vendargues et St-Jean-de-Védas paraissent être politiquement «parties prenantes» dans le district, la première étant un territoire plus industriel, la seconde se développant à partir du commerce concentré qui dessert l'agglomération.

Les deux offrent les traits des modalités nouvelles du développement local (tableau 4, p. 95), soit un taux de toutes petites industries plus élevé que l'ensemble, tout en étant celles qui accueillent les plus grandes entreprises, un taux d'activité des services aux entreprises très important et une croissance des emplois révélant le dynamisme del'activitéćconomique. Les deux semblent allerd'un même mouvement, guidées par le district vers la nouvelle «structure-projet» de la région. Les deux communes ont choisi des maires originaires du village.

L'évolution économique du Crès se fait aussi au rythme du développement du District, mais cette commune qui sait faire fructifier les retombées du rayonnement montpelliérain ne s'engage pas activement dans cette structure de regroupement et diversifie ses stratégies d'appartenance.

La commune de Juvignac tente de se donner une spécificité en bloquant le développement de sa zone industrielle pour construire une image de «territoirepoumon" par l'aménagement d'une zone verte de loisirs et la remise en exploitation de sources thermales.

Ces différenciations dans les politiques communales semblent souvent être élaborées en référence à la politique du District et plus précisément aux projets du député maire de Montpellier. 
En dehors des clivages liés aux appartenances politiques, des résistances se construisent sur la crainte d'une perte d'autonomie et d'identité. Et il ne suffit pas de parler d'esprit de clocher pour comprendre ce phénomène. En fait, ce qui est à l'œuvre dans chaque communec'est une conception spécifique des modes de gestion et de transformation du territoire portée par les élus et/ou une partie active de la population. Ces villes du District sont menacées de perdre ou de ne pouvoir reconquérir une identité dans une banalisation du territoire qui doit porter un processus uniforme de développement selon les critères de la modernité et les ambitions de la capitale. Il est nécessaire alors de construire un «territoire-projet» propre accompagnant (complétant ou s'opposant) le «grand» territoire-projet du District. Et c'est ce que semblent faire, de façon plus ou moins explicite, les élus des différentes communes observées.

Ici on pense son territoire comme porteur de luxe, de repos et de santé,... communes dortoirs qui vont retrouver un standing et développer la plus-value immobilière; là on accepte la rapide extension des zones d'activités et on restaure un centre de village où on installe des activités culturelles; plus loin on s'applique à imposer aux promoteurs immobiliers la reproduction des places de village chaque fois qu'un nouvel espace loti se construit; ailleurs on applique au territoire différentes stratégies de regroupement selon les services à créer ou à étendre. Chaque «territoireprojet» prend son originalité sous l'impulsion de maires qui, pour les plus actifs, construisent leur propre image de marque en construisant celle de leur commune.

L'origine locale de certains d'entre eux leur a donné des atouts pour gérer l'explosion démographique de leur commune sans rupture avec les autochtones, pour promouvoir le sol communal et distribuer la manne selon les inégalités et les rapports de force existant (entre couches sociales) avant le mouvement d'urbanisation.

Les communes rurales, elles, n'ont pas eu dans l'ensemble l'opportunité de reconstruire une originalité ou de se réapproprier le territoire. Elles ont tenté de l'offrir dans une politique vitrine, et de séduire des preneurs mais à un moment où déjà la capitale proche absorbait PME et PMI performantes.

Cependant par une politique authentique de développement endogène, de mâitrise du territoire-construit, la commune de St-Mathieu-de-Tréviers, par son profil économique, ressemble fort aux communes les plus intégrées dans le District, mais se distingue par la précocité de l'élaboration d'une politique autonome et de promotion de son territoire-projet. Sous l'impulsion du Maire, des mesures ont été prises pour que les permis de construire soient accordés en fonction de la croissance du potentiel économique local c'est-à-dire des emplois. Commune rurale, StMathieu a cherché à préserver la viticulture par une politique de vin de qualité et a pu attirer des petites industries grâce à un cadre physique séduisant et l'aménagement d'une zone industrielle à la fin des années 60 .

La politique reste axée sur un développement des emplois plus que sur la recherche de la taxe professionnelle. Mais le dynamisme industriel commence à être 
freiné par la concurrence de l'agglomération urbaine, et des stratégies de diversification des activités sont élaborées en accord avec les communes environnantes afin que la masse critique soit suffisante pour rentabiliser des installations sportives et de loisirs chargées de soutenir et de renforcer la dynamique de cette zone au nord de Montpellier.

L'exemple de St-Mathieu est précieux parce qu'il a innové dans le mode de mise en présence de deux types de partenaires: les chefs entreprises et les élus locaux. Conscient de la nécessité d'un développement par la base, le maire de la commune a fait en sorte que la population soit concernée. Le «territoire-projet» proposé rendait actifs dans la démarche tous les partenaires potentiels.

Les élus s'ouvraient à un réel dialogue avec les créateurs d'entreprises postulants; ceux-ci étaient intéressés par des «politiques» moins traditionnelles.

Ceci a préfiguré la transformation en œuvre locale de la mise en rapport de deux logiques : celle de l'entreprise privée qui cherche les meilleures conditions de rentabilisation de ses investissements et celle d'un pouvoir politique qui cherche à satisfaire les besoins des électeurs pour perdurer.

Par rapport au territoire, tout se passait la plupart du temps comme si se rencontraient dans le meilleur des cas, deux conceptions du territoire ou deux types de relations au territoire :

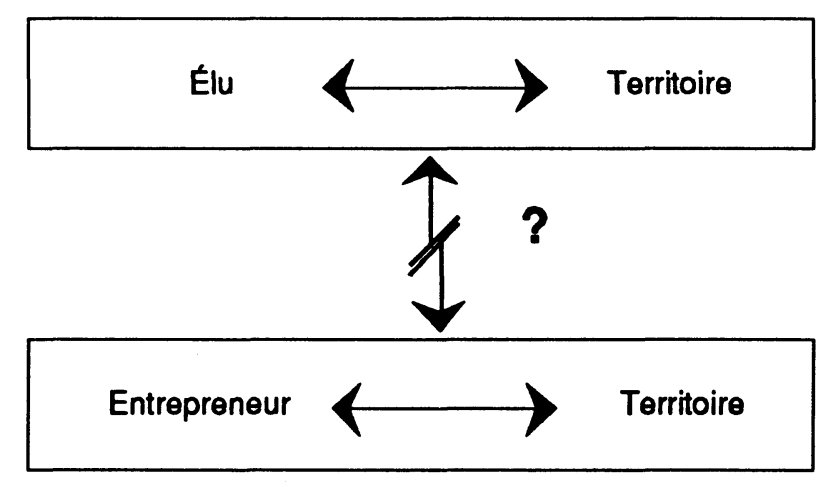

l'une caractérisée par l'absence de projet collectif et un conservatisme qui ne tolérait guère que la spéculation immobilière, aubaine pour une économie de monoculture en perte de vitesse; l'autre fondée bien souvent sur la seule recherche de profit se traduisant par des implantations d'entreprises (ou d'établissements de grandes entreprises) qui n'avaient pas toujours de perspective d'insertion locale durable.

A la faveur de l'émergence du rôle de la petite entreprise et de la multiplication des PME en Languedoc, la relation entre l'élu et le responsable de l'entreprise ou l'innovateur a pu changer de nature. La petite taille de l'entreprise favorise le contact, 
et le patron d'une petite entreprise est plus accessible que le PDG d'une multinationale. De son côté, l'élu est pris dans le changement culturel qui consiste depuis quelques années à revaloriser l'entreprise et son rôle social.

La relation se construit à partir de conceptions compatibles sur le devenir d'un territoire, d'autant plus que le développement actuel de l'économie locale se fait à partir des activités de service non polluantes qui permettent de protéger et valoriser le cadre naturel et de satisfaire les populations habituées à une certaine qualité de l'environnement.

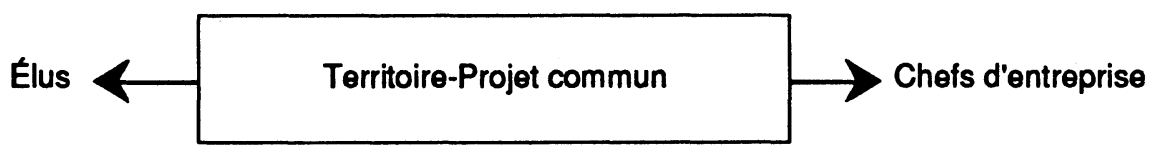

Par ailleurs, les entrepreneurs qui s'installent actuellement dans la région cherchent les partenariats et les synergies qui ne peuvent se structurer que dans un territoire agi à la fois par les agents du développement local, les élus, les chambres syndicales etc.

Au total, il semblerait que dans cette relation le responsable politique ait fait le premier pas et le chemin le plus long. Le responsable d'entreprise reste avant tout un preneur de territoire qui n'a pas souvent le temps d'investir le domaine politique et d'accepter une charge d'élu. Seuls quelques artisans sont conseillers municipaux dans les communes observées (quelques responsables connus de grandes entreprises sont tentés par l'aventure politique et se constituent une notabilité dans quelques villes de grande ou moyenne importance)

Mais un partage des tâches, les uns s'occupant du collectif public, les autres du collectif entreprise, ne crée pas forcément d'opposition et ce d'autant moins que les communes attirent des investisseurs motivés par un cadre de vie et type de valorisation de l'espace que la population et les responsables locaux entendent conserver et préserver.

Il reste à rappeler la différence de dynamisme qui existe maintenant sur le territoire étudié entre une technopole qui risque de s'isoler des environnements socio-économiques régionaux (Gilly, 1987)et des communes qui doivent trouver les moyens et le souffle d'un développement autonome. 
Tab. 4. Profil des Communes

\begin{tabular}{|c|c|c|c|c|c|}
\hline & $\begin{array}{c}1 \\
\text { Emploi \% }\end{array}$ & $\begin{array}{c}2 \\
\mathrm{PI} \leq 10 \% \\
\end{array}$ & $\begin{array}{c}3 \\
\text { \% Sonvices } \\
\text { aux entreprises }\end{array}$ & $\begin{array}{c}4 \\
\text { PME-PML10\% } \\
\end{array}$ & \begin{tabular}{|c|}
5 \\
\% d'entreprises \\
créos entre 85 ot 87 \\
\end{tabular} \\
\hline$\left[\begin{array}{c}\text { St -Georges- } \\
\text { d'Orques }\end{array}\right.$ & 34,4 & 5 & 13,3 & 93,3 & 58,3 \\
\hline Juvignac & 20,1 & 7 & 8 & 85,8 & 61,44 \\
\hline S.Total & 24,2 & 6,3 & 10 & 88,6 & 61 \\
\hline Le Crès & 1,2 & 7,4 & 7,4 & 93,8 & 64,8 \\
\hline Vendargues & 32,85 & 13,2 & 33,3 & 82,7 & 60,9 \\
\hline $\begin{array}{l}\text { St Jean- } \\
\text { de - Védas }\end{array}$ & 36 & 7,97 & 15,2 & 81,8 & 66 \\
\hline$\left[\begin{array}{l}\text { Clermont- } \\
\text { L'Hérault }\end{array}\right.$ & 13,1 & 2,5 & 7,1 & 93,5 & 47,3 \\
\hline $\begin{array}{l}\text { St-André- } \\
\text { de-Sangonis }\end{array}$ & 10 & 9,2 & 3,9 & 100 & 48,05 \\
\hline S.Total & 12,7 & 3,9 & 6,47 & 94,9 & 47,6 \\
\hline $\begin{array}{l}\text { St- Mathieu- } \\
\text { de-Tréviers }\end{array}$ & 25,6 & 14,8 & 17,1 & 91,4 & 71,6 \\
\hline $\begin{array}{l}\text { Le -Grau- } \\
\text { du -Roi }\end{array}$ & - & 4,13 & 13,9 & 95,7 & 48,7 \\
\hline Ensemble & 23,83 & 6,63 & 13,74 & 92,9 & 55 \\
\hline
\end{tabular}

(1) Coefficient de croissance des emplois $(1985,1986)$ qui n'a de valeur que pour la comparaison entre les communes.

(2) Pourcentage, sur l'effectif total d'entreprises, des industries de petite taille.

(3) Ensemble des entreprises $\leq 10$ salariés : part dans l'effectif total 


\section{BIBLIOGRAPHIE}

Cognée, I. et Vernazobres, P., (1985), «Le rôle des structures intercommunales dans l'implantation d'entreprises», ERFI, Montpellier.

Gilly, J.P., (1987), «Innovation et Territoire : Pour une approche socio-économique des technopoles». Revue d' Économie Régionale et Urbaine,n'5.

Soulage, B., (1987), «Le développement local endogène, possibilités et limites». Revue d'Économie Régionale et Urbaine, $\mathrm{n}^{\circ} 3$.

Uhrich, R., (1987), La France inverse?, Economica, Paris. 


\section{Annexe 1}

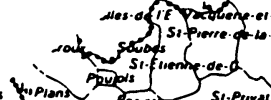

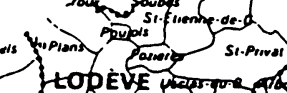

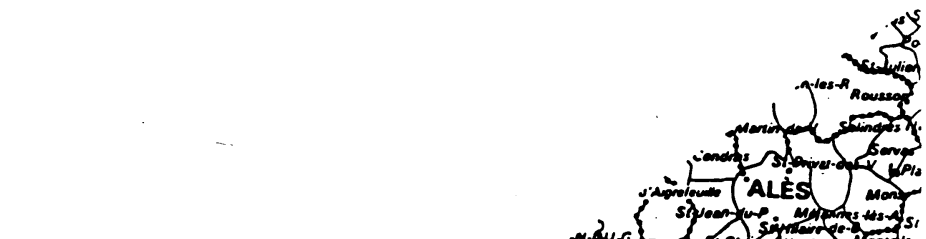

The

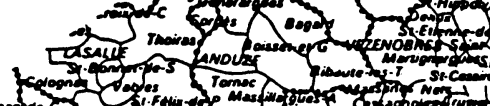

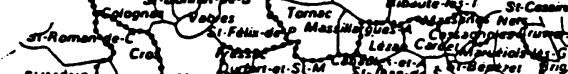

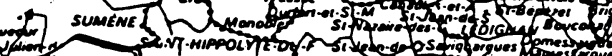

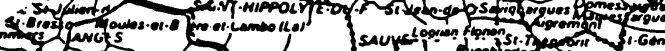

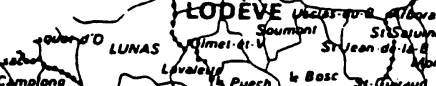

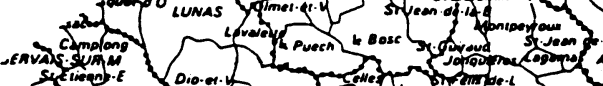

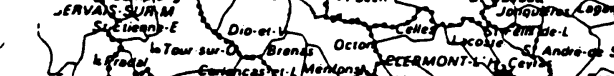
Thessectioi comber the tone

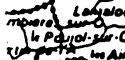

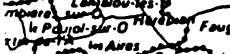

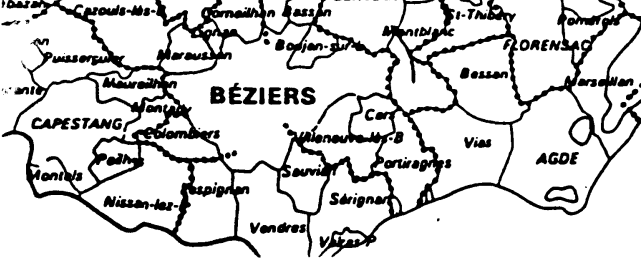

